\title{
A Meta-Analysis of the Association between DNMT1 Polymorphisms and Cancer Risk
}

\author{
Hao Li, Jing-wei Liu, Li-ping Sun, and Yuan Yuan \\ Tumor Etiology and Screening Department, Cancer Institute and General Surgery, \\ The First Affiliated Hospital of China Medical University, Key Laboratory of Cancer Etiology and Prevention \\ (China Medical University), Liaoning Provincial Education Department, Shenyang, Liaoning 110001, China \\ Correspondence should be addressed to Li-ping Sun; lpsun@cmu.edu.cn and Yuan Yuan; yuanyuan@cmu.edu.cn
}

Received 29 November 2016; Accepted 20 March 2017; Published 3 April 2017

Academic Editor: Jinsong Ren

Copyright (C) 2017 Hao Li et al. This is an open access article distributed under the Creative Commons Attribution License, which permits unrestricted use, distribution, and reproduction in any medium, provided the original work is properly cited.

Previous studies have examined the associations of DNA methyltransferase 1 (DNMT1) polymorphisms, including single nucleotide polymorphisms rs16999593 (T/C), rs2228611 (G/A), and rs2228612 (A/G), with cancer risk. However, the results are inconclusive. The aim of this meta-analysis is to elucidate the associations between DNMT1 polymorphisms and cancer susceptibility. The PubMed, Embase, Web of Science, and Chinese National Knowledge Infrastructure databases were searched systematically to identify potentially eligible reports. Odd ratios and 95\% confidence intervals were used to evaluate the strength of association between three DNMT1 polymorphisms and cancer risk. A total of 16 studies were finally included in the meta-analysis, namely, nine studies of 3378 cases and 4244 controls for rs16999593, 11 studies of 3643 cases and 3866 controls for rs2228611, and three studies of 1343 cases and 1309 controls for rs2228612. The DNMT1 rs2228612 (A/G) polymorphism was significantly related to cancer risk in the recessive model. The meta-analysis also suggested that DNMT1 rs16999593 (T/C) may be associated with gastric cancer, while rs2228611 (G/A) may be associated with breast cancer. In future research, large-scale and well-designed studies are required to verify these findings.

\section{Introduction}

DNA methylation is one of the most commonly occurring epigenetic events in the mammalian genome. DNA methyltransferases (DNMTs) are critical to establishing and maintaining DNA methylation patterns by converting cytosine residues to 5-methylcytosine $(5 \mathrm{mC})$ in cytosine-guanine (CpG) dinucleotides $[1,2]$. The DNMT family comprises three active forms: DNMT1, DNMT3A, and DNMT3B. DNMT1 is the major enzyme responsible for methylation maintenance $[3,4]$, while DNMT3A and DNMT3B are thought to function in de novo methylation rather than in methylation maintenance $[3,4]$.

DNMT1 is located on human chromosome 19p13.2 and encodes a protein comprising 1632 amino acids, which may be implicated in occurrence progression and prognosis of the cancer. DNMT1 consists of three major structural elements: an $\mathrm{N}$-terminal regulatory domain that is necessary for the localization of DNMT1; a C-terminal catalytic domain that is involved in the binding of substrates; and a central linker that contains repeated glycine-lysine dipeptides [5]. Genetic variation caused by single nucleotide polymorphisms (SNPs) is the most common form of altered gene structure. The most commonly studied DNMT1 SNPs are rs16999593 (T/C), rs2228611 (G/A), and rs2228612 (A/G), which are present in coding regions and, therefore, may influence DNMT1 expression.

In recent years, various studies have indicated that DNMT1 polymorphisms may play pivotal roles in carcinogenesis. The SNPs rs16999593 (T/C) and rs2228612 (G/A) were initially found to be associated with risk of breast cancer $[6,7]$, while SNP rs2228611 (A/G) was linked to gastric cancer [8]. Subsequently, a number of studies have concentrated on the relationships between DNMT1 polymorphisms and risks of different cancers [6, 7, 9-21]. However, the results from these studies are inconsistent. 
Until now, no meta-analysis has been carried out to investigate the relation of the three DNMT1 polymorphisms (rs16999593 (T/C), rs2228611 (G/A), and rs2228612 (A/G)) with risk of cancer. Individual studies have lacked the ability to obtain overall reliable conclusions because of limited sample sizes and/or variations in ethnicities. To obtain further insights into the roles of DNMT1 polymorphisms in carcinogenesis, we mainly performed a meta-analysis on the associations between these three SNPs and cancer risk.

\section{Materials and Methods}

2.1. Search Strategy. We systematically searched the PubMed, Embase, Web of Science, and Chinese National Knowledge Infrastructure databases using different combinations of the search terms "DNMT1 or DNA methyltransferase 1," "polymorphism or mutation or variant," and "cancer or neoplasm or tumor." The search was last updated on 06 June 2016. When overlapping data were found, only the largest and latest study was selected. We contacted the authors and requested their specific raw data when the data provided in the published article were not sufficient.

2.2. Inclusion and Exclusion Criteria. Studies were included when they met the following criteria: case-control study; the subject was the association of DNMT1 polymorphisms (rs16999593 (T/C), rs2228611 (G/A), and rs2228612 (A/G)) with risk of cancer; and essential information on genotype or allele frequencies was available to assess the odds ratios (ORs) and 95\% confidence intervals (CIs). Exclusion criteria included review articles; systematic reviews and metaanalyses; animal studies; sample size less than 100; and scarce or insufficient information on genotype or allele frequencies for the rs16999593 (T/C), rs2228611 (G/A), or rs2228612 $(\mathrm{A} / \mathrm{G})$ polymorphisms of DNMT1 despite us contacting the authors.

2.3. Data Extraction. Two of the authors (HL and JL) independently selected the articles and extracted the original data using a standardized and consistent method. The following information was collected from each study: first author, year of publication, ethnicity of the subjects, cancer type, numbers of cases and controls, and genotyping methods. Conflicts were resolved after discussion and consensus was finally reached on all the extracted information.

2.4. Statistical Analysis. All statistical analyses were conducted using STATA software (version 12.0; Stata Corp LP, College Station, TX). ORs and their corresponding 95\% CIs were employed to assess the strength of relationships between the DNMT1 polymorphisms and cancer risk. $P$ values $<0.05$ were considered as statistically significant. Heterogeneity was calculated using the $Q$ statistic $(P$ value $<$ 0.10 indicates significant heterogeneity among studies) and $I$ squared value. The Mantel-Haenszel fixed-effects model was used to calculate the pooled ORs when the heterogeneity of studies was not significant. Otherwise, the DerSimonian and Laird random-effects model was used. We conducted the sensitivity analysis to explore heterogeneity when significant heterogeneity existed. Subgroup analysis was applied to explore the effects of cancer type and genotyping method. In addition, Begg's test and Egger's test were performed to evaluate publication bias; $P$ values $<0.05$ for Begg's and Egger's tests indicate significant publication bias.

\section{Results}

3.1. Characteristics of the Studies. This meta-analysis was organized according to the PRISMA (Supplementary File 1 in Supplementary Material available online at https://doi .org/10.1155/2017/3971259). A detailed flow chart of the study selection process is shown in Figure 1. A total of 215 potentially relevant articles were found by searching the four databases and after removing duplicates. Altogether 187 publications were excluded mainly due to no relevance, animal not human experiments, reviews, or meeting abstract. The 28 remaining articles were evaluated further for eligibility. Finally, 16 articles were included in the present meta-analysis [6-21].

The baseline characteristics of the 16 included studies are summarized in Table 1. Among them, nine articles containing 3378 cases and 4244 controls surveyed the association between rs16999593 (T/C) and cancer risk [6, 7, 9-15]; 11 studies on the relation between $\mathrm{rs} 2228611$ (G/A) and cancer risk included 3643 cases and 3866 controls [6$8,10,11,13,14,18-21]$; and three publications containing 1343 cases and 1309 controls explored the correlation of rs2228612 (A/G) with cancer risk [7, 16, 17]. The populations surveyed in the nine rs16999593 (T/C) studies were all Chinese [6, 7, 9-15]. In the rs2228611 (G/A) studies, nine of the populations were Chinese $[6,7,10,11,13,14,19-21]$, one was Iranian [8], and one was Polish [18]. In the rs2228612 $(\mathrm{A} / \mathrm{G})$ studies the populations were either Caucasian [16] or Chinese $[7,17]$. The genotyping methods used to detect the DNMT1 polymorphisms included sequencing, MassARRAY, PCR-RFLP, MALDI-TOF, TaqMan, and SNPlex [6-21]. We used subgroup analyses to explore the effects of different cancer types and genotyping methods on the associations of increased risk of cancer with the DNMT1 rs16999593 (T/C) and rs2228611 (G/A) polymorphisms. We did not perform subgroup analysis for rs2228612 (A/G) because of the limited number of articles that was available.

3.2. Quantitative Data Synthesis. The results for the association of DNMT1 rs16999593 (T/C) with cancer risk are summarized in Table 2. Overall, neither the heterozygous nor dominant genetic models found significant associations between rs16999593 (T/C) and cancer risk (TC versus TT: OR $=1.29,95 \% \mathrm{CI}=0.90-1.84, P=0.163$; TC $+\mathrm{CC}$ versus TT: $\mathrm{OR}=1.28,95 \% \mathrm{CI}=0.93-1.77, P=0.135)$. The allele analysis also found no significant association (C allele versus $\mathrm{T}$ allele: $\mathrm{OR}=1.18,95 \% \mathrm{CI}=0.96-1.45, P=0.127)$. For the subgroup analysis according to cancer type (Figure 2), rs16999593 (T/C) was consistently associated with increased risk of gastric cancer (TC versus TT: $\mathrm{OR}=1.36,95 \% \mathrm{CI}=1.14-1.61, P=$ 0.001; $\mathrm{TC}+\mathrm{CC}$ versus TT: $\mathrm{OR}=1.36,95 \% \mathrm{CI}=1.15-1.60$, 


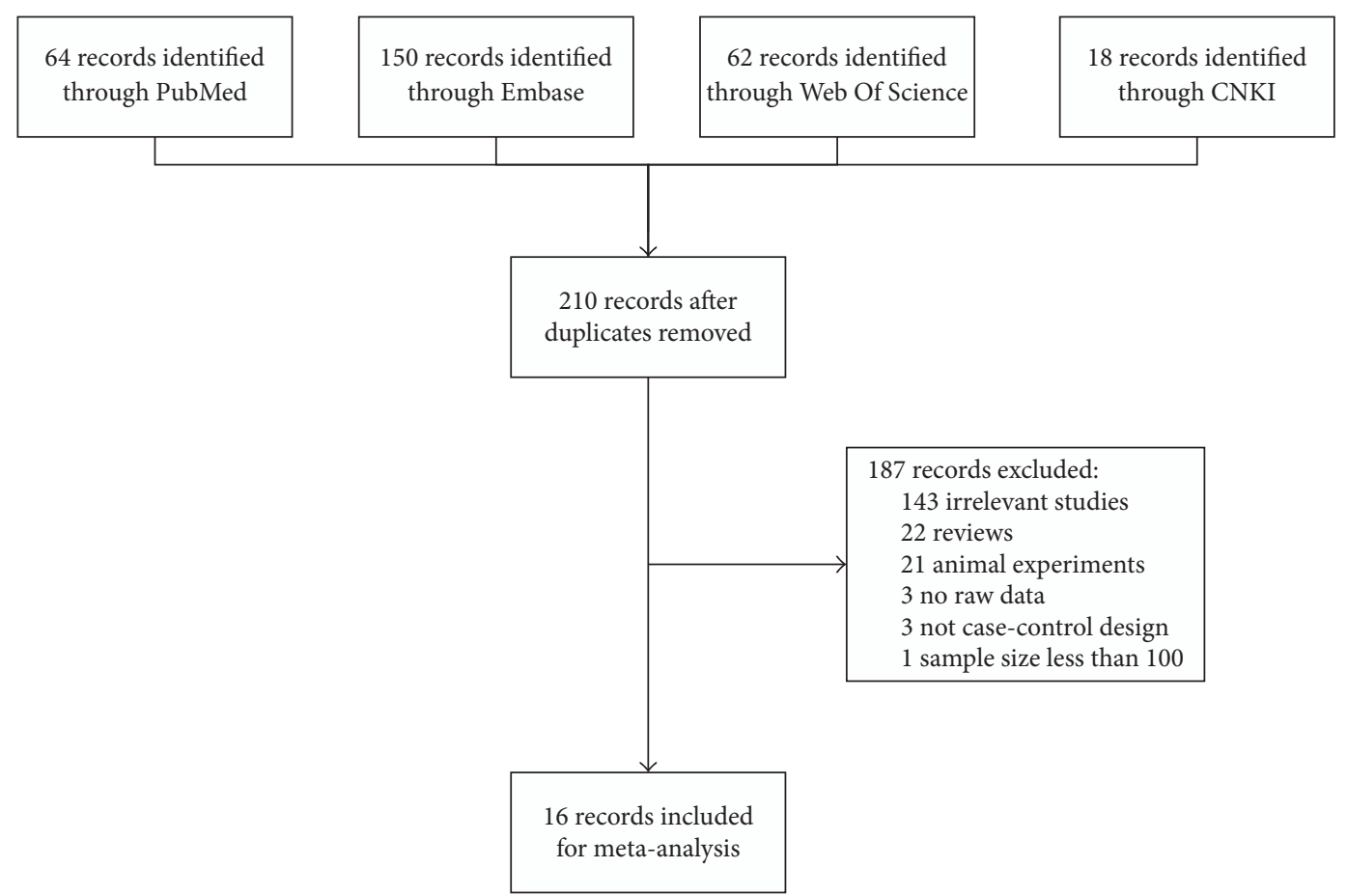

FIGURE 1: The flowchart of literature inclusion and exclusion.

TABLE 1: Characteristics of the included studies in this meta-analysis.

\begin{tabular}{|c|c|c|c|c|c|c|c|c|c|c|c|c|}
\hline \multirow{2}{*}{ Author } & \multirow{2}{*}{ Year } & \multirow{2}{*}{ Ethnicity } & \multirow{2}{*}{ Cancer type } & \multirow{2}{*}{ Genotyping method } & \multicolumn{4}{|c|}{ Case } & \multicolumn{4}{|c|}{ Control } \\
\hline & & & & & Total & MM & WM & WW & Total & MM & WM & WW \\
\hline \multicolumn{13}{|c|}{ For DNMT1 rs16999593 T/C polymorphism } \\
\hline Wang [12] & 2014 & Chinese & Cervical cancer & Sequencing & 100 & 48 & 44 & 8 & 100 & 70 & 25 & 5 \\
\hline Gao [13] & 2015 & Chinese & Gastric cancer & Sequencing & 310 & 180 & 112 & 18 & 420 & 281 & 117 & 22 \\
\hline $\mathrm{Li}[14]$ & 2015 & Chinese & Esophageal cancer & MassARRAY & 258 & 138 & 80 & 40 & 260 & 127 & 109 & 24 \\
\hline He et al. [11] & 2014 & Chinese & Prostate cancer & MassARRAY & 155 & 94 & 53 & 8 & 155 & 73 & 67 & 15 \\
\hline Sun [7] & 2012 & Chinese & Breast cancer & MassARRAY & 1327 & 425 & 224 & 29 & 1440 & 504 & 202 & 28 \\
\hline Xiang et al. [6] & 2010 & Chinese & Breast cancer & PCR-RFLP & 305 & 239 & 64 & 2 & 314 & 220 & 89 & 5 \\
\hline Jiang et al. [9] & 2012 & Chinese & Gastric cancer & Sequencing & 447 & 283 & 144 & 20 & 961 & 659 & 273 & 29 \\
\hline Yang et al. [10] & 2012 & Chinese & Gastric cancer & MALDI-TOF & 242 & 141 & 89 & 12 & 294 & 196 & 83 & 15 \\
\hline & 2015 & Chinese & Breast cancer & Sequencing & 234 & 68 & 164 & 2 & 300 & 180 & 105 & 15 \\
\hline \multicolumn{13}{|c|}{ For DNMT1 rs2228611 G/A polymorphism } \\
\hline Gao [13] & 2015 & Chinese & Gastric cancer & Sequencing & 310 & 167 & 128 & 15 & 420 & 232 & 163 & 25 \\
\hline $\mathrm{Li}[14]$ & 2015 & Chinese & Esophageal cancer & MassARRAY & 258 & 131 & 85 & 42 & 260 & 119 & 113 & 28 \\
\hline He et al. [11] & 2014 & Chinese & Prostate cancer & Mass & 155 & 82 & 61 & 12 & 155 & 79 & 64 & 12 \\
\hline Xiang et al. [6] & 2010 & Chinese & Breast cancer & LP & 305 & 125 & 149 & 31 & 314 & 154 & 121 & 39 \\
\hline Yang et al. [21] & 2016 & Chinese & Renal cell carcinoma & PCR- & 293 & 152 & 117 & 24 & 293 & 139 & 133 & 21 \\
\hline Yang et al. [10] & 2012 & Chinese & Gastric cancer & MALDI-TOF & 242 & 132 & 97 & 13 & 285 & 160 & 99 & 26 \\
\hline Sun [7] & 2012 & Chinese & Breast cancer & MassARRAY & 678 & 341 & 279 & 58 & 733 & 369 & 303 & 61 \\
\hline Mostowska et al. [18] & 2013 & Polish & Ovarian cancer & PCR-RFLP & 159 & 28 & 74 & 57 & 210 & 44 & 94 & 72 \\
\hline Xi et al. [19] & 2014 & Chinese & Breast cancer & MALDI-TOF & 810 & 385 & 362 & 63 & 848 & 432 & 343 & 73 \\
\hline Lin et al. [20] & 2015 & Chinese & Breast cancer & MALDI-TOF & 233 & 107 & 109 & 17 & 236 & 120 & 94 & 22 \\
\hline Khatami et al. [8] & 2009 & Iranian & Gastric cancer & PCR-RFLP & 200 & 34 & 50 & 16 & 112 & 32 & 62 & 18 \\
\hline \multicolumn{13}{|c|}{ For DNMT1 rs 2228612 A/G polymorphism } \\
\hline Sun [7] & 2012 & Chinese & Breast cancer & MassARRAY & 675 & 254 & 273 & 148 & 731 & 308 & 290 & 133 \\
\hline Chang et al. [17] & 2014 & Chinese & Esophageal cancer & SNPlex & 137 & 52 & 56 & 29 & 357 & 100 & 200 & 57 \\
\hline Chang et al. [17] & 2014 & Chinese & Stomach cancer & SNPlex & 143 & 43 & 72 & 28 & 357 & 100 & 200 & 57 \\
\hline Chang et al. [17] & 2014 & Chinese & Liver cancer & SNPlex & 158 & 48 & 74 & 36 & 357 & 100 & 200 & 57 \\
\hline Kullmann et al. [16] & 2013 & Caucasian & Breast cancer & TaqMan & 221 & 193 & 28 & 0 & 221 & 180 & 35 & 6 \\
\hline
\end{tabular}

Abbreviations: W, wild-type allele; M, mutant-type allele. 


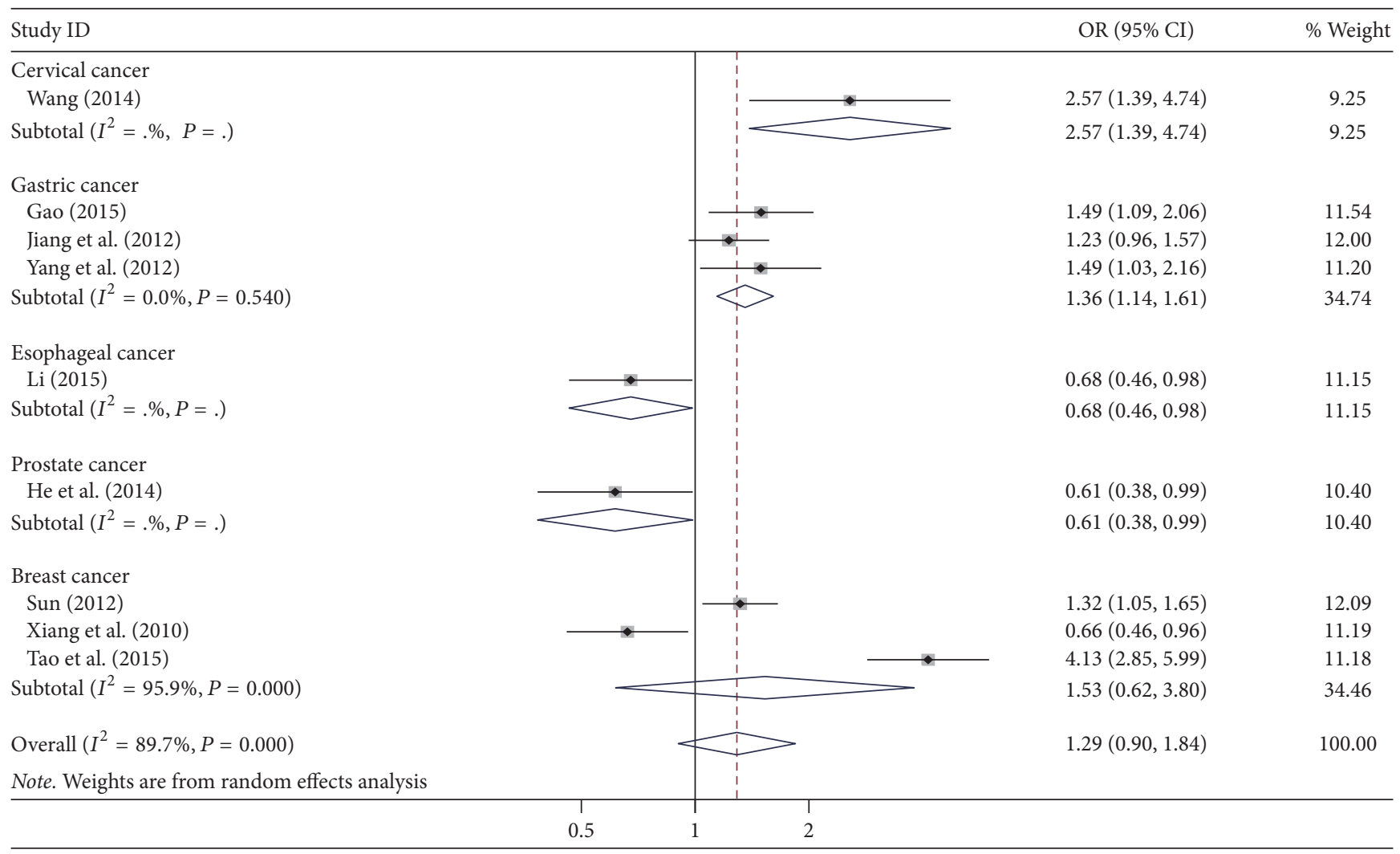

(a)

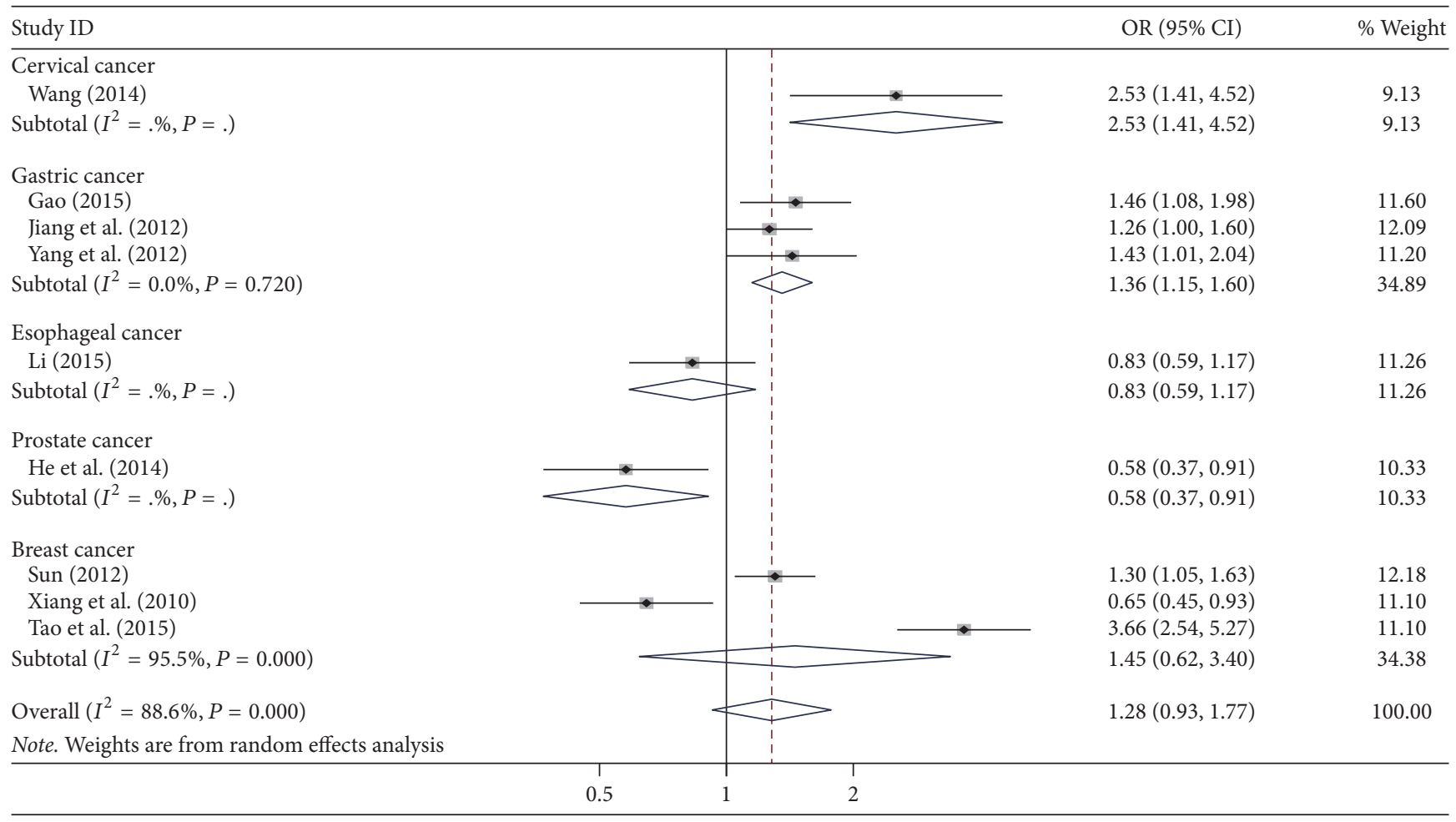

(b)

FIGURE 2: Forest plot for the association between DNMT1 rs16999593 (T/C) polymorphism and cancer risk in the cancer type subgroup. (a) TC versus TT; (b) TC + CC versus TT. 
TABLE 2: Meta-analysis results of the association between DNMT1 rs16999593 (T/C) polymorphism and cancer risk.

\begin{tabular}{|c|c|c|c|c|c|c|c|}
\hline \multirow{2}{*}{ Genetic model } & \multirow{2}{*}{ Group/subgroup } & \multirow{2}{*}{$N$} & \multicolumn{2}{|c|}{ Heterogeneity test } & \multirow{2}{*}{ Statistical model } & \multicolumn{2}{|c|}{ Test for overall effect } \\
\hline & & & $I_{2}(\%)$ & $P_{\text {het }}$ & & OR $(95 \% \mathrm{CI})$ & $P$ \\
\hline \multirow{5}{*}{ CC versus TT } & Overall & 9 & 37.5 & 0.119 & $\mathrm{~F}$ & $1.17(0.92-1.49)$ & 0.213 \\
\hline & Gastric cancer & 3 & 0.0 & 0.743 & $\mathrm{~F}$ & $1.36(0.93-1.99)$ & 0.117 \\
\hline & Breast cancer & 3 & 48.1 & 0.146 & $\mathrm{~F}$ & $0.93(0.58-1.48)$ & 0.748 \\
\hline & Sequencing & 4 & 30.1 & 0.232 & $\mathrm{~F}$ & $1.33(0.90-1.95)$ & 0.149 \\
\hline & MassARRAY & 3 & 66.1 & 0.052 & $\mathrm{R}$ & $1.01(0.53-1.93)$ & 0.968 \\
\hline \multirow{5}{*}{ TC versus TT } & Overall & 9 & 89.70 & $<0.001$ & $\mathrm{R}$ & $1.29(0.90-1.84)$ & 0.163 \\
\hline & Gastric cancer & 3 & 00.00 & 0.540 & $\mathrm{~F}$ & $1.36(1.14-1.61)$ & 0.001 \\
\hline & Breast cancer & 3 & 95.90 & $<0.001$ & $\mathrm{R}$ & $1.53(0.62-3.80)$ & 0.360 \\
\hline & Sequencing & 4 & 90.30 & $<0.001$ & $\mathrm{R}$ & $2.06(1.16-3.65)$ & 0.013 \\
\hline & MassARRAY & 3 & 85.40 & 0.001 & $\mathrm{R}$ & $0.84(0.49-1.43)$ & 0.517 \\
\hline \multirow{5}{*}{$(\mathrm{TC}+\mathrm{CC})$ versus TT } & Overall & 9 & 88.60 & $<0.001$ & $\mathrm{R}$ & $1.28(0.93-1.72)$ & 0.135 \\
\hline & Gastric cancer & 3 & 00.00 & 0.720 & $\mathrm{~F}$ & $1.36(1.15-1.60)$ & $<0.001$ \\
\hline & Breast cancer & 3 & 95.50 & $<0.001$ & $\mathrm{R}$ & $1.45(0.62-3.40)$ & 0.388 \\
\hline & Sequencing & 4 & 88.40 & $<0.001$ & $\mathrm{R}$ & $1.99(1.20-3.28)$ & 0.007 \\
\hline & MassARRAY & 3 & 83.60 & 0.002 & $\mathrm{R}$ & $0.88(0.55-1.41)$ & 0.603 \\
\hline \multirow{5}{*}{$\mathrm{CC}$ versus $(\mathrm{TC}+\mathrm{TT})$} & Overall & 9 & 48.7 & 0.049 & $\mathrm{R}$ & $1.03(0.72-1.49)$ & 0.861 \\
\hline & Gastric cancer & 3 & 0.00 & 0.635 & $\mathrm{~F}$ & $1.22(0.84-1.78)$ & 0.303 \\
\hline & Breast cancer & 3 & 70.5 & 0.034 & $\mathrm{R}$ & $0.49(0.13-1.76)$ & 0.274 \\
\hline & Sequencing & 4 & 62.9 & 0.044 & $\mathrm{R}$ & $1.00(0.49-2.04)$ & 0.998 \\
\hline & MassARRAY & 3 & 65.8 & 0.054 & $\mathrm{R}$ & $1.10(0.59-2.05)$ & 0.767 \\
\hline \multirow{5}{*}{$\mathrm{C}$ allele versus $\mathrm{T}$ allele } & Overall & 9 & 81.40 & $<0.001$ & $\mathrm{R}$ & $1.18(0.95-1.45)$ & 0.127 \\
\hline & Gastric cancer & 3 & 00.00 & 0.936 & $\mathrm{~F}$ & $1.28(1.11-1.47)$ & 0.001 \\
\hline & Breast cancer & 3 & 91.50 & $<0.001$ & $\mathrm{R}$ & $1.18(0.71-1.97)$ & 0.529 \\
\hline & Sequencing & 4 & 66.30 & 0.030 & $\mathrm{R}$ & $1.53(1.26-1.95)$ & $<0.001$ \\
\hline & MassARRAY & 3 & 81.10 & 0.005 & $\mathrm{R}$ & $0.96(0.67-1.36)$ & 0.805 \\
\hline
\end{tabular}

Abbreviations: R, random effect model; F, fixed effect model.

$P<0.001 ; \mathrm{C}$ allele versus $\mathrm{T}$ allele: $\mathrm{OR}=1.28,95 \% \mathrm{CI}=$ $1.11-1.47, P=0.001)$, but no significant association was found with breast cancer. For the different genotyping methods, rs16999593 (T/C) demonstrated increased risk of cancer in the sequencing subgroup but not in the MassARRAY subgroup.

The results for the association of DNMT1 rs2228611 (G/A) with cancer risk are summarized in Table 3. Overall, the GA genotype was not significantly associated with risk of cancer compared with the GG genotype (OR $=1.05,95 \% \mathrm{CI}$ $=0.92-1.21, P=0.075)$, and the GA + AA genotype was not related to cancer risk compared with the GG genotype $(\mathrm{OR}=$ $1.05,95 \% \mathrm{CI}=0.96-1.15, P=0.284)$. Similarly, no significant association was observed in the allele analysis (A allele versus G allele: $\mathrm{OR}=1.02,95 \% \mathrm{CI}=0.95-1.10, P=0.532)$. In the subgroup analysis according to cancer type (Figure 3 ), the rs2228611 (G/A) was associated with higher risk of breast cancer (GA versus GG: OR $=1.17,95 \% \mathrm{CI}=1.03-1.33, P=$ 0.016 ; $\mathrm{GA}+\mathrm{AA}$ versus $\mathrm{GG}$ : $\mathrm{OR}=1.13,95 \% \mathrm{CI}=1.00-1.28$, $P=0.043)$. A similar result was found in the MALDI-TOF subgroup (GA versus GG: $\mathrm{OR}=1.20,95 \% \mathrm{CI}=1.03-1.41$, $P=0.022$ ) in the analysis according to different genotyping methods. For the gastric cancer and PCR-RFLP subgroups, no significant associations were found in any of the compared genetic models.
The results for the association of DNMT1 rs2228612 (A/G) with cancer risk are summarized in Table 4 and Figure 4. Significant association with cancer risk was observed in the recessive model (GG versus $\mathrm{AG}+\mathrm{AA}$ : $\mathrm{OR}=1.29,95 \% \mathrm{CI}$ $=1.06-1.56, P=0.009)$, but no significant association was revealed in the allele analysis ( $G$ allele versus $A$ allele: $O R=$ $1.00,95 \% \mathrm{CI}=0.83-1.20, P=0.980)$.

3.3. Heterogeneity Test and Sensitivity Analysis. In most of the comparisons of DNMT1 rs16999593 and rs2228612 polymorphisms and one comparison of DNMT1 rs2228611, significant heterogeneity was observed. We next performed a leave-one-out sensitivity analysis. The results show that no individual study significantly affected the pooled OR (figure not shown), suggesting that the results of the meta-analysis were robust.

3.4. Publication Bias. Begg's test and Egger's test were used to quantitatively evaluate the publication bias of the selected studies; the details are listed in Table 5. For the associations of DNMT1 rs16999593 (T/C), rs2228611 (G/A), and rs2228612 $(\mathrm{A} / \mathrm{G})$ with cancer risk, rs2228612 (A/G) showed publication bias (GG versus AA; G allele versus A allele) and rs16999593 $(\mathrm{T} / \mathrm{C})$ showed publication bias in the comparison with the recessive model. 


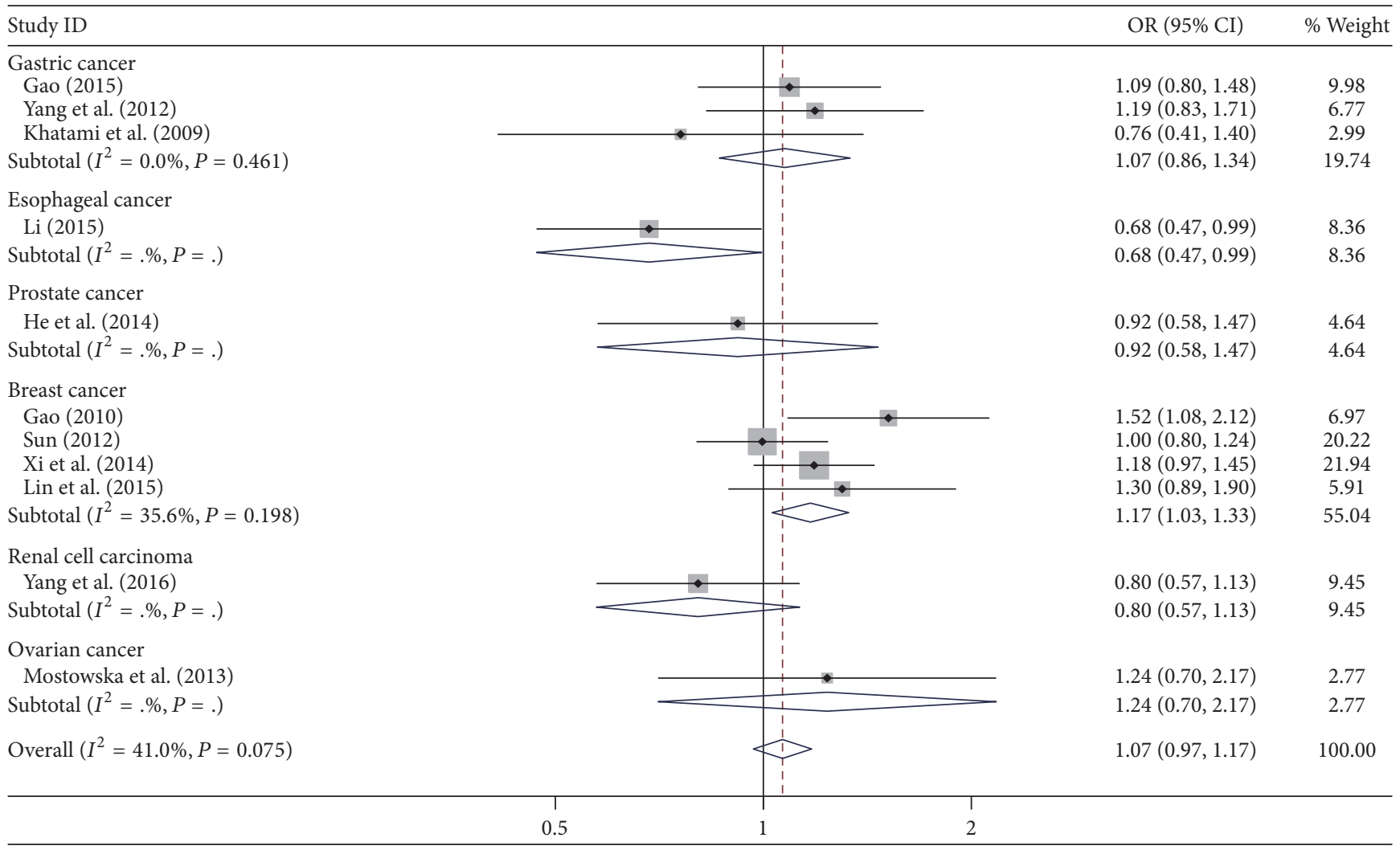

(a)

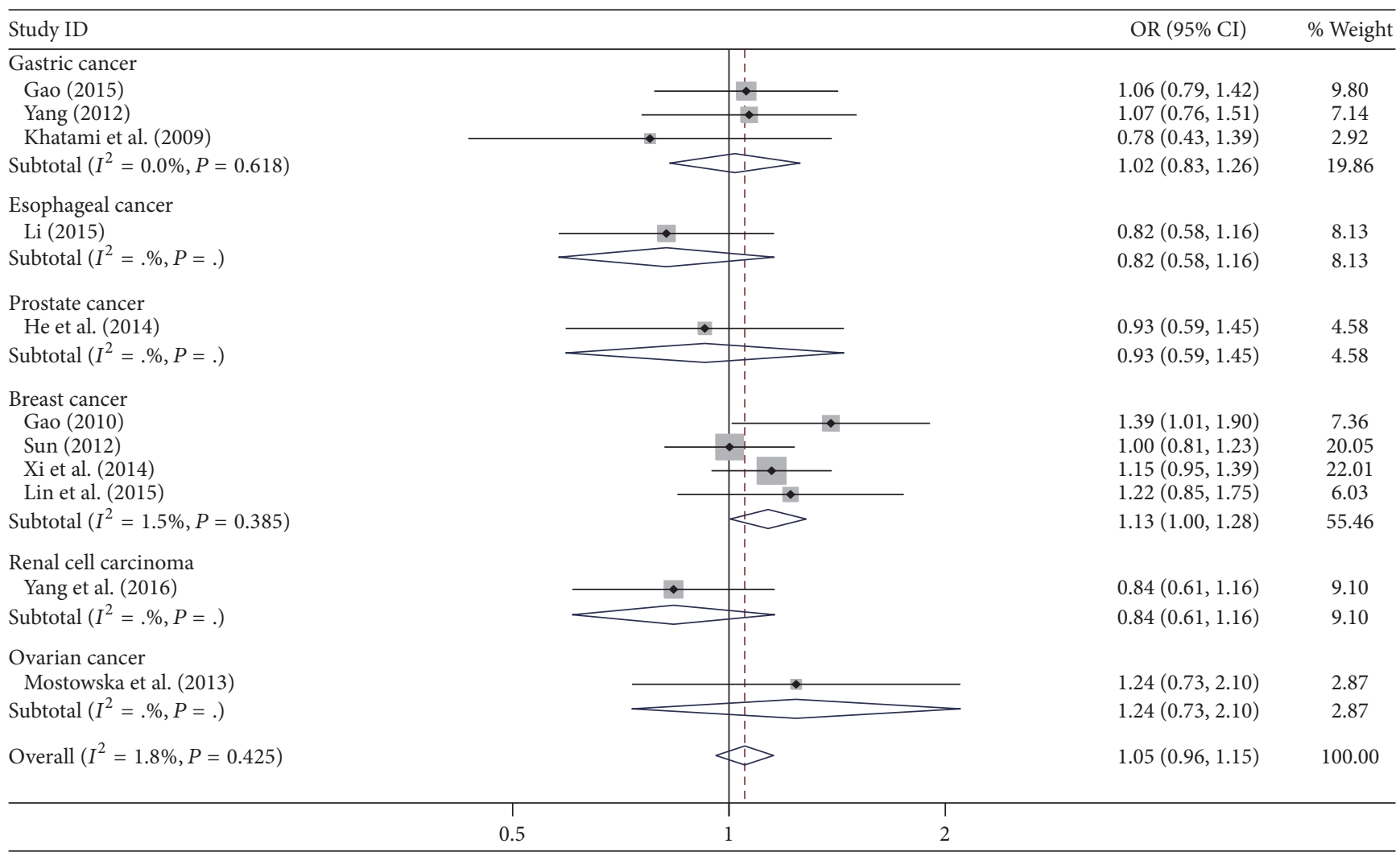

(b)

FIGURE 3: Forest plot for the association between DNMT1 rs2228611 (G/A) polymorphism and risk of cancer in the subgroup of cancer type. (a) GA versus GG; (b) GA + AA versus GG. 
TABLE 3: Meta-analysis results of the association between DNMT1 rs2228611 (G/A) polymorphism and cancer risk.

\begin{tabular}{|c|c|c|c|c|c|c|c|}
\hline \multirow{2}{*}{ Genetic model } & \multirow{2}{*}{ Group/subgroup } & \multirow{2}{*}{ Studies } & \multicolumn{2}{|c|}{ Heterogeneity test } & \multirow{2}{*}{ Statistical model } & \multicolumn{2}{|c|}{ Test for overall effect } \\
\hline & & & $I_{2}(\%)$ & $P_{\text {het }}$ & & OR (95\% CI) & $P$ \\
\hline \multirow{5}{*}{ AA versus GG } & Overall & 11 & 0.00 & 0.925 & $\mathrm{~F}$ & $0.99(0.84-1.19)$ & 0.898 \\
\hline & Gastric cancer & 3 & 0.00 & 0.774 & $\mathrm{~F}$ & $0.74(0.49-1.13)$ & 0.165 \\
\hline & Breast cancer & 4 & 0.00 & 0.979 & $\mathrm{~F}$ & $0.98(0.78-1.22)$ & 0.848 \\
\hline & PCR-RFLP & 4 & 0.00 & 0.880 & $\mathrm{~F}$ & $1.04(0.76-1.42)$ & 0.803 \\
\hline & MALDI-TOF & 3 & 0.00 & 0.511 & $\mathrm{~F}$ & $0.87(0.65-1.17)$ & 0.360 \\
\hline \multirow{5}{*}{ GA versus GG } & Overall & 11 & 41.00 & 0.075 & $\mathrm{R}$ & $1.05(0.92-1.21)$ & 0.445 \\
\hline & Gastric cancer & 3 & 0.00 & 0.461 & $\mathrm{~F}$ & $1.07(0.86-1.33)$ & 0.522 \\
\hline & Breast cancer & 4 & 35.60 & 0.198 & $\mathrm{~F}$ & $1.17(1.03-1.33)$ & 0.016 \\
\hline & PCR-RFLP & 4 & 64.00 & 0.040 & $\mathrm{R}$ & $1.05(0.73-1.52)$ & 0.782 \\
\hline & MALDI-TOF & 3 & 0.00 & 0.910 & $\mathrm{~F}$ & $1.20(1.03-1.41)$ & 0.022 \\
\hline \multirow{5}{*}{$(\mathrm{GA}+\mathrm{AA})$ versus $\mathrm{GG}$} & Overall & 11 & 1.80 & 0.425 & $\mathrm{~F}$ & $1.05(0.96-1.15)$ & 0.284 \\
\hline & Gastric cancer & 3 & 0.00 & 0.618 & $\mathrm{~F}$ & $1.02(0.83-1.26)$ & 0.860 \\
\hline & Breast cancer & 4 & 1.50 & 0.385 & $\mathrm{~F}$ & $1.13(1.00-1.28)$ & 0.043 \\
\hline & PCR-RFLP & 4 & 51.70 & 0.102 & $\mathrm{~F}$ & $1.06(0.87-1.29)$ & 0.542 \\
\hline & MALDI-TOF & 3 & 0.00 & 0.872 & $\mathrm{~F}$ & $1.14(0.98-1.33)$ & 0.087 \\
\hline \multirow{5}{*}{ AA versus $(\mathrm{GA}+\mathrm{GG})$} & Overall & 11 & 0.00 & 0.596 & F & $0.97(0.83-1.13)$ & 0.671 \\
\hline & Gastric cancer & 3 & 0.00 & 0.535 & $\mathrm{~F}$ & $0.76(0.51-1.12)$ & 0.169 \\
\hline & Breast cancer & 4 & 0.00 & 0.813 & $\mathrm{~F}$ & $0.90(0.73-1.12)$ & 0.351 \\
\hline & PCR-RFLP & 4 & 0.00 & 0.779 & $\mathrm{~F}$ & $0.99(0.76-1.29)$ & 0.933 \\
\hline & MALDI-TOF & 3 & 0.00 & 0.501 & $\mathrm{~F}$ & $0.80(0.60-1.06)$ & 0.126 \\
\hline \multirow{5}{*}{ A allele versus $G$ allele } & Overall & 11 & 0.00 & 0.978 & $\mathrm{~F}$ & $1.02(0.95-1.10)$ & 0.532 \\
\hline & Gastric cancer & 3 & 0.00 & 0.863 & $\mathrm{~F}$ & $0.96(0.82-1.14)$ & 0.660 \\
\hline & Breast cancer & 4 & 0.00 & 0.861 & $\mathrm{~F}$ & $1.06(0.96-1.16)$ & 0.249 \\
\hline & PCR-RFLP & 4 & 0.00 & 0.530 & $\mathrm{~F}$ & $1.03(0.90-1.18)$ & 0.696 \\
\hline & MALDI-TOF & 3 & 0.00 & 0.745 & $\mathrm{~F}$ & $1.04(0.93-1.17)$ & 0.494 \\
\hline
\end{tabular}

R: random effect model; F: fixed effect model.

TABLE 4: Meta-analysis results of the association between DNMT1 rs2228612 (A/G) polymorphism and cancer risk.

\begin{tabular}{lcccc}
\hline \multirow{2}{*}{ Genetic model } & \multicolumn{2}{c}{ Heterogeneity test } & Statistical model & \multicolumn{2}{c}{ Test for overall effect } \\
& $I_{2}(\%)$ & $P_{\text {het }}$ & OR (95\% CI) & $1.20(0.97-1.49)$ \\
\hline GG versus AA & 19.20 & 0.292 & F & $0.081(0.61-1.08)$ \\
AG versus AA & 60.30 & 0.039 & R & 0.156 \\
(GG + AG) versus AA & 62.7 & 0.030 & $\mathrm{R}$ & $0.87(0.66-1.14)$ \\
GG versus (AA + AG) & 11.50 & 0.340 & $\mathrm{~F}$ & $\mathbf{1 . 2 9}(\mathbf{1 . 0 6}-\mathbf{1 . 5 6})$ \\
G allele versus A allele & 58.70 & 0.046 & $\mathrm{R}$ & $\mathbf{0 . 0 0 9}$ \\
\hline
\end{tabular}

R: random effect model; F: fixed effect model.

\section{Discussion}

DNMT1, the major methyltransferase in mammals, lies in the replication fork and methylates newly synthesized DNA strands directly in S phase of DNA replication sites [22], which is essential for epigenetic inheritance. Regional aberrant DNA hypermethylation has been identified as a possible mechanism of inactivation of tumor suppressor genes [23]. Many studies have indicated that the overexpression of DNMT1 could silence vital tumor suppressor genes such as $A P C, P 16$, and RUNX3 through DNA methylation [24, 25]. Therefore, DNMT1 might be implicated in the occurrence, development, and prognosis of multiple types of cancer.
Polymorphisms have been identified as a powerful tool for predicting hereditary susceptibility of some complex diseases including cancer. However, previous individual studies about the association between DNMT1 polymorphisms and cancer risk were not only limited but also inconclusive. To our knowledge, this is the first comprehensive meta-analysis investigating the possible correlations of SNPs rs16999593 (T/C), rs2228611 (G/A), and rs2228612 (A/G) in DNMT1 with risk of overall cancer and specific cancer types, which is anticipated to shed light on the role of DNMT1 polymorphisms in carcinogenesis.

SNP rs2228612 (A/G) causes an isoleucine to phenylalanine substitution at amino acid 327 in the DNMT1 protein, 


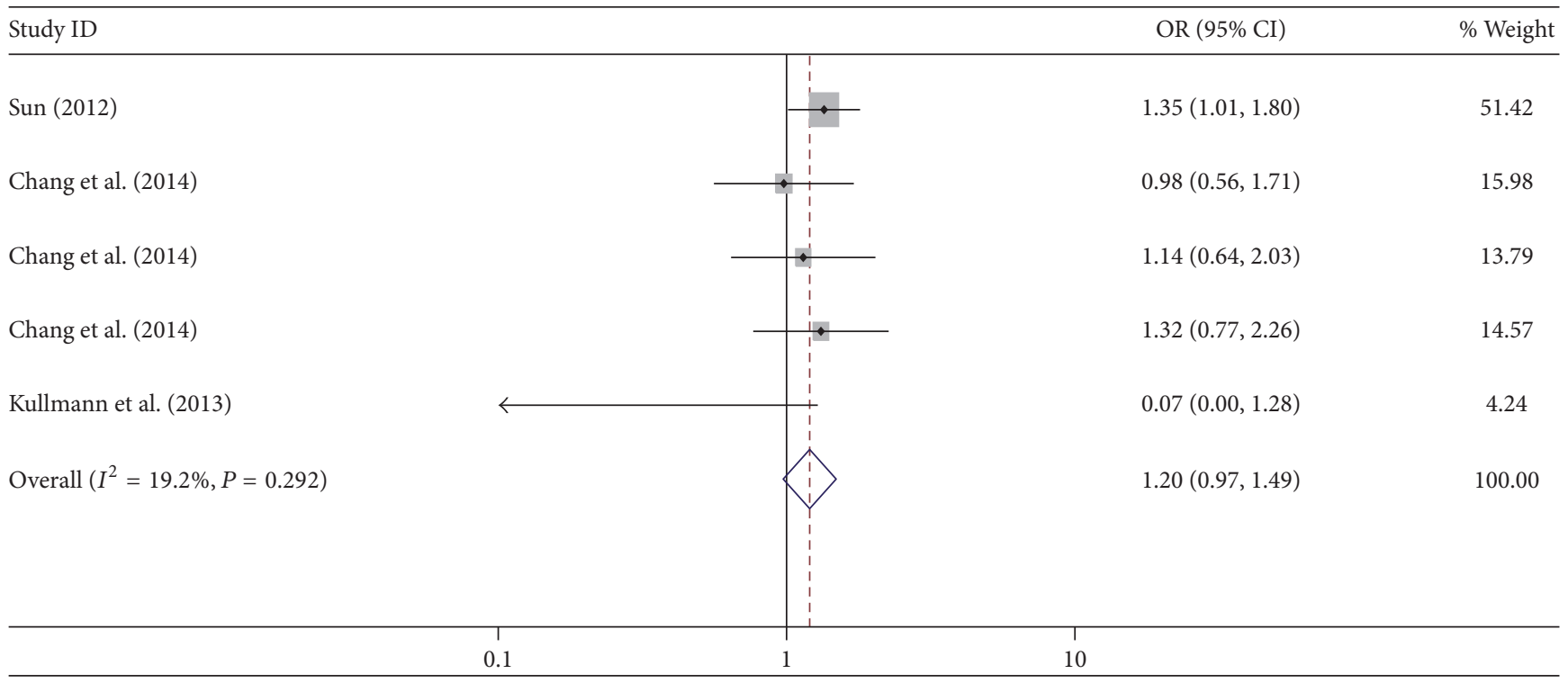

(a)

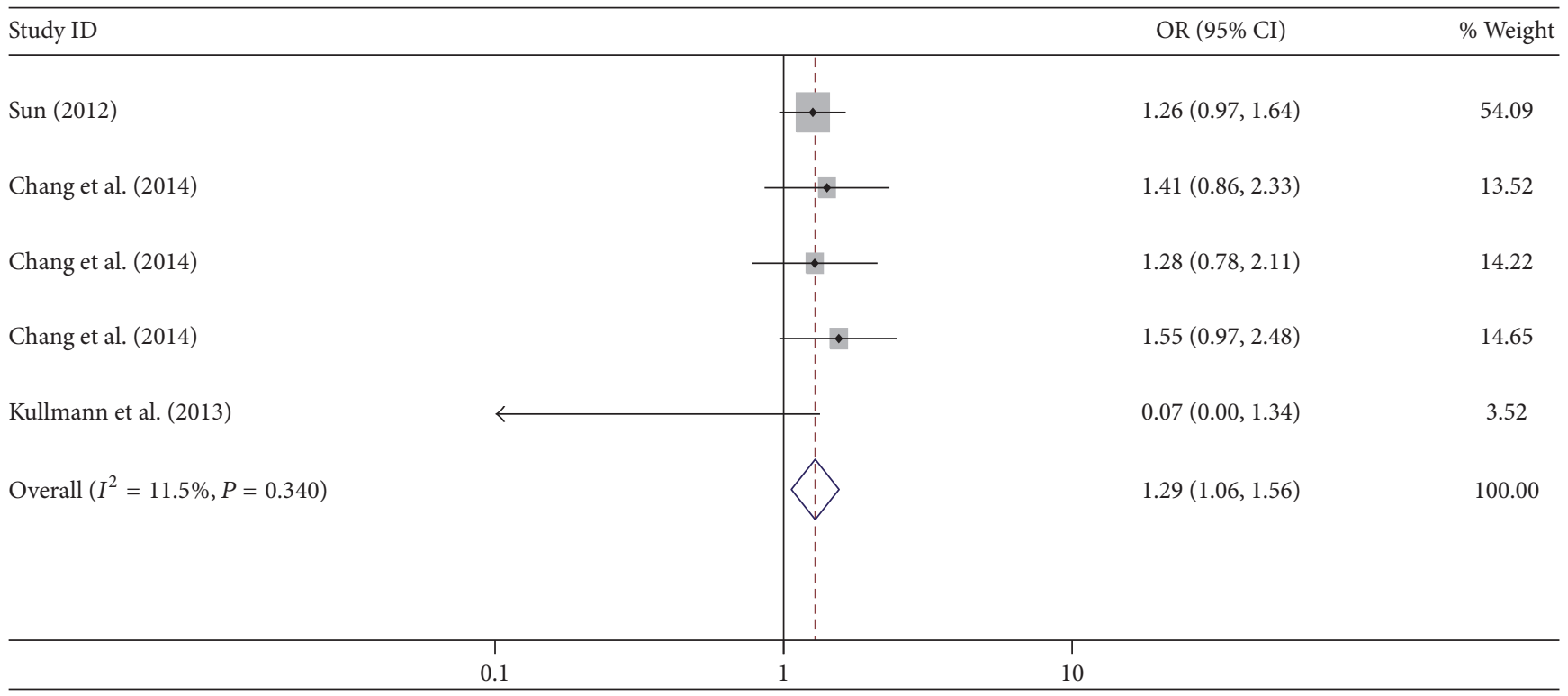

(b)

FIGURE 4: Forest plot for the association between DNMT1 rs2228612 (A/G) polymorphism and cancer risk. (a) GG versus AA; (b) GG versus $\mathrm{AA}+\mathrm{AG}$.

which may alter the function of DNMT1 and influence its effect in the carcinogenesis. In this meta-analysis, we found that DNMT1 rs2228612 (A/G) was associated with risk of overall cancer in the recessive model. However, only three articles with small-scale studies were available for analysis; therefore, the results should to be interpreted with caution.

We did not find significant association between SNP rs16999593 (T/C) and overall cancer risk in any genetic comparison. Different types of cancer have distinct initiation and progression mechanisms, in which polymorphisms in key genes play critical roles. This meta-analysis elucidated that the DNMT1 rs16999593 (T/C) polymorphism was associated with different cancer types. In the subgroup analysis according to cancer type, the TC genotype of rs16999593 (T/C) was associated with risk of gastric cancer, but not breast cancer. Extensive evidence has suggested that DNA methylation is involved in the initiation and progression of gastric cancer and increased expression of DNMT1 had been confirmed to be related to gastric cancer [25]. The AKT$\mathrm{NF} \kappa \mathrm{B}$ and STAT3 signaling pathways can upregulate DNMT1 expression, which could cause aberrant DNA methylation of tumor suppressor genes and lead to gastric cancer [26, 27]. Therefore, SNP rs16999593 (T/C), which causes a histidine to arginine substitution at 97 positions of the amino acid 
TABLE 5: Results of publication bias test.

\begin{tabular}{|c|c|c|c|c|c|}
\hline \multirow{2}{*}{ Polymorphism } & \multirow{2}{*}{ Compared genotype } & \multicolumn{2}{|c|}{ Begg's test } & \multicolumn{2}{|c|}{ Egger's test } \\
\hline & & $z$-value & $P$ value & $t$-value & $P$ value \\
\hline \multirow{5}{*}{ DNMT1 rs16999593 T/C } & CC versus TT & 0.94 & 0.348 & -2.00 & 0.085 \\
\hline & TC versus TT & 0.10 & 0.917 & 0.03 & 0.976 \\
\hline & $(\mathrm{TC}+\mathrm{CC})$ versus TT & 0.10 & 0.917 & 0.02 & 0.982 \\
\hline & CC versus $(\mathrm{TC}+\mathrm{TT})$ & 1.98 & 0.048 & -2.72 & 0.030 \\
\hline & $\mathrm{C}$ allele versus $\mathrm{T}$ allele & 0.10 & 0.917 & -0.45 & 0.669 \\
\hline \multirow{5}{*}{ DNMT1 rs2228611 G/A } & AA versus GG & 1.40 & 0.161 & -0.99 & 0.348 \\
\hline & GA versus GG & 0.78 & 0.436 & -0.65 & 0.530 \\
\hline & $(\mathrm{GA}+\mathrm{AA})$ versus $\mathrm{GG}$ & 0.78 & 0.436 & -0.69 & 0.505 \\
\hline & AA versus (GA + GG) & 0.47 & 0.640 & -0.61 & 0.556 \\
\hline & A allele versus $G$ allele & 0.93 & 0.350 & 1.17 & 0.273 \\
\hline \multirow{5}{*}{ DNMT1 rs2228612 (A/G) } & GG versus AA & 1.71 & 0.086 & -3.97 & 0.029 \\
\hline & AG versus AA & 0.24 & 0.806 & -2.86 & 0.065 \\
\hline & $(G G+G A)$ versus $A A$ & 0.73 & 0.462 & -3.81 & 0.032 \\
\hline & GG versus $(\mathrm{AA}+\mathrm{AG})$ & 0.73 & 0.462 & -1.37 & 0.263 \\
\hline & G allele versus A allele & 2.20 & 0.027 & -4.26 & 0.024 \\
\hline
\end{tabular}

sequence, might affect the function of DNMT1, thus increasing susceptibility to gastric cancer. The subgroup of breast cancer involved two types of breast cancer: sporadic triplenegative breast cancer (TNBC) [15] and infiltrating ductal beast carcinoma (IDBC) [6]. The TC genotype of rs16999593 (T/C) was related to increased TNBC risk but decreased IDBC risk. Therefore, DNMT1 might have diverse functions in different types of breast cancer. Further studies of the effects of DNMT1 polymorphisms on specific breast cancer types are still needed. In addition, only in the subgroup of sequencing, DNMT1 rs16999593 (T/C) were constantly associated with increased cancer risk in all compared genetic models, indicating that different genotype detecting methods might influence the results.

According to the functional prediction tool F-SNP (http://compbio.cs.queensu.ca/F-SNP/), SNP rs2228611 (G/A) may change the regulation of DNMT1 splicing by leading to a synonymous mutation at amino acid 463 . Multiple transcript variants of DNMT1 gene as a result of alternative splicing have been found. Therefore, we speculated that the rs2228611 (G/A) might influence the process of carcinogenesis by regulating the pattern of alternative splicing of DNMT1. Here, we did not find any significant associations between DNMT1 rs2228611 (G/A) with altered risk of cancer in any genetic model. However, in the subgroup analysis, individuals with the GA genotype of rs2228611 (G/A) were associated with higher risk of breast cancer in both heterozygous and dominant models. In addition, individuals with the GA genotype of rs2228611 (G/A) had decreased risk in one study on esophageal cancer [14], which was opposite to the results for breast cancer. This reverse outcome may be because various types of cancer have different mechanisms of carcinogenesis. Future studies on different types of cancer may help to better understand these findings.

Some limitations of our meta-analysis should be noted. Firstly, the number of studies was not sufficiently large, especially for subgroup analysis of DNMT1 rs2228612 (A/G). Secondly, the languages of the publications were limited to English and Chinese. Thirdly, although this meta-analysis was based on the whole population, most studies were from Chinese populations, except for one Iranian case and one Polish case for rs2228611 (G/A) and another one Caucasian case for rs2228612 (A/G), which showed the same results with the Chinese population. So this study had a certain universality, especially for Chinese population. Finally, publication bias was found in two comparisons of rs2228612 (A/G) and one comparison of rs16999593 (T/C).

\section{Conclusion}

The DNMT1 rs2228612 (A/G) GG genotype may be associated with increased risk of cancer compared with the AA + AG genotype. SNP rs16999593 (T/C) is significantly associated with gastric cancer risk while SNP rs2228611 (G/A) is significantly related to breast cancer risk. Further large-scale and well-designed investigations including different cancers are required to verify the findings of this study.

\section{Disclosure}

The authors alone are responsible for the content and writing of the paper.

\section{Conflicts of Interest}

The authors declare that they have no conflicts of interest.

\section{Authors' Contributions}

Hao Li and Jing-wei Liu performed statistical analysis and data interpretation and wrote the paper. Li-ping Sun and 
Yuan Yuan conceived this study and revised the manuscript. All authors read and approved the final manuscript.

\section{Acknowledgments}

This study was supported by the National Key R\&D Program, Grant 2016YFC1303200, and the Key Laboratory of Cancer Intervention in Liaoning Province, Grant LS201605.

\section{References}

[1] K. D. Robertson, "DNA methylation, methyltransferases, and cancer," Oncogene, vol. 20, no. 24, pp. 3139-3155, 2001.

[2] A. Razin and A. D. Riggs, "DNA methylation and gene function," Science, vol. 210, no. 4470, pp. 604-610, 1980.

[3] M. W. Łuczak and P. P. Jagodziński, "The role of DNA methylation in cancer development," Folia Histochemica et Cytobiologica, vol. 44, no. 3, pp. 143-154, 2006.

[4] M. T. McCabe, J. C. Brandes, and P. M. Vertino, "Cancer DNA methylation: molecular mechanisms and clinical implications," Clinical Cancer Research, vol. 15, no. 12, pp. 3927-3937, 2009.

[5] J. Turek-Plewa and P. P. Jagodziński, “The role of mammalian DNA methyltransferases in the regulation of gene expression," Cellular and Molecular Biology Letters, vol. 10, no. 4, pp. 631-647, 2005.

[6] G. Xiang, F. Zhenkun, C. Shuang et al., "Association of DNMT1 gene polymorphisms in exons with sporadic infiltrating ductal breast carcinoma among chinese han women in the heilongjiang province," Clinical Breast Cancer, vol. 10, no. 5, pp. 373-377, 2010.

[7] M. Y. Sun, Association study of single nucleotide polymorphisms in estrogen synthesis, metabolism-related genes, and DNMTs with the susceptibility of breast cancer among Han Chinese women [M.S. thesis], Nanfang Medical University, Guangzhou, China, 2012.

[8] F. Khatami, B. Noorinayer, S. Ghiasi, R. Mohebi, M. Hashemi, and M. R. Zali, "Lack of effects of single nucleotide polymorphisms of the DNA methyltransferase 1 gene on gastric cancer in Iranian patients: a case control study," Asian Pacific Journal of Cancer Prevention, vol. 10, no. 6, pp. 1177-1182, 2009.

[9] J. Jiang, Z. Jia, D. Cao et al., "Polymorphisms of the DNA methyltransferase 1 associated with reduced risks of helicobacter pylori infection and increased risks of gastric atrophy," PLoS ONE, vol. 7, no. 9, Article ID e46058, 2012.

[10] X.-X. Yang, X.-Q. He, F.-X. Li, Y.-S. Wu, Y. Gao, and M. Li, "Risk-association of DNA methyltransferases polymorphisms with gastric cancer in the Southern Chinese population," International Journal of Molecular Sciences, vol. 13, no. 7, pp. 83648378, 2012.

[11] B.-S. He, Y.-Q. Pan, and C.-B. Zhu, "Polymorphisms of DNA methyltransferases and the risk of prostate cancer," Zhonghua Nan Ke Xue, vol. 20, no. 12, pp. 1077-1081, 2014.

[12] L. Wang, "Correlation of DNA methyctransferase 1 gene polymorphisms and passive smoking with cervical cancer," International Journal of Gynecology \& Obstetrics, no. 6, pp. 663666, 2014.

[13] Y. Gao, "Association of Dnmt 1 single-mucleotide polymorphisms and risk of gastric caner," Chinese Journal of Cancer Prevention and Treatment, no. 6, pp. 394-397, 2015.
[14] H. Li, "Association of polymorphisms of DNMT1 and DNMT3B with risk of esophageal cancer," Labeled Immunoassays and Clinical Medicine, no. 12, pp. 1214-1220, 2015.

[15] R. Tao, Z. Chen, P. Wu et al., "The possible role of EZH2 and DNMT1 polymorphisms in sporadic triple-negative breast carcinoma in southern Chinese females," Tumor Biology, vol. 36, no. 12, pp. 9849-9855, 2015.

[16] K. Kullmann, M. Deryal, M. F. Ong, W. Schmidt, and U. Mahlknecht, "DNMT1 genetic polymorphisms affect breast cancer risk in the central European Caucasian population," Clinical Epigenetics, vol. 5, no. 1, article 7, 2013.

[17] S.-C. Chang, P.-Y. Chang, B. Butler et al., "Single nucleotide polymorphisms of one-carbon metabolism and cancers of the esophagus, stomach, and liver in a Chinese population," PLoS ONE, vol. 9, no. 10, Article ID e109235, 2014.

[18] A. Mostowska, S. Sajdak, P. Pawlik, M. Lianeri, and P. P. Jagodzinski, "DNMT1, DNMT3A and DNMT3B gene variants in relation to ovarian cancer risk in the Polish population," Molecular Biology Reports, vol. 40, no. 8, pp. 4893-4899, 2013.

[19] J. Xi, Y. Su, A. B. Fadiel et al., "Association of physical activity and polymorphisms in FGFR2 and DNA methylation related genes with breast cancer risk," Cancer Epidemiology, vol. 38, no. 6, pp. 708-714, 2014.

[20] W. Lin, Y. L. Cen, Y. Lin et al., "Joint effects between urinary selenium and polymorphisms in methylation related genes on breast cancer risk," Neoplasma, vol. 62, no. 3, pp. 491-499, 2015.

[21] S.-M. Yang, C.-Y. Huang, H.-S. Shiue et al., "Combined effects of DNA methyltransferase 1 and $3 \mathrm{~A}$ polymorphisms and urinary total arsenic levels on the risk for clear cell renal cell carcinoma," Toxicology and Applied Pharmacology, vol. 305, pp. 103-110, 2016.

[22] C. J. Klein, M.-V. Botuyan, Y. Wu et al., "Mutations in DNMT1 cause hereditary sensory neuropathy with dementia and hearing loss," Nature Genetics, vol. 43, no. 6, pp. 595-600, 2011.

[23] S. B. Baylin, M. Makos, J. Wu et al., "Abnormal patterns of DNA methylation in human neoplasia: potential consequences for tumor progression," Cancer Cells, vol. 3, no. 10, pp. 383-390, 1991.

[24] C. A. Eads, A. E. Nickel, and P. W. Laird, "Complete genetic suppression of polyp formation and reduction of CPG-island hypermethylation in ApcMin/+ Dnmtl-hypomorphic mice," Cancer Research, vol. 62, no. 5, pp. 1296-1299, 2002.

[25] W. Chen, N. Gao, Y. Shen, and J.-N. Cen, "Hypermethylation downregulates Runx3 gene expression and its restoration suppresses gastric epithelial cell growth by inducing p27 and caspase 3 in human gastric cancer," Journal of Gastroenterology and Hepatology (Australia), vol. 25, no. 4, pp. 823-831, 2010.

[26] B.-G. Zhang, L. Hu, M.-D. Zang et al., "Helicobacter pylori CagA induces tumor suppressor gene hypermethylation by upregulating DNMT1 via AKT-NF $\kappa$ B pathway in gastric cancer development," Oncotarget, vol. 7, no. 9, pp. 9788-9800, 2016.

[27] Q. Zhang, H. Y. Wang, A. Woetmann, P. N. Raghunath, N. Odum, and M. A. Wasik, "STAT3 induces transcription of the DNA methyltransferase 1 gene (DNMT1) in malignant T lymphocytes," Blood, vol. 108, no. 3, pp. 1058-1064, 2006. 


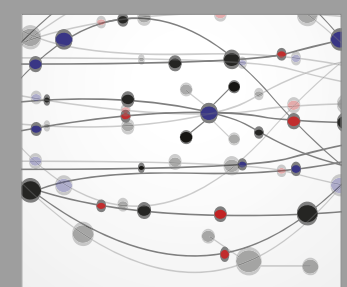

The Scientific World Journal
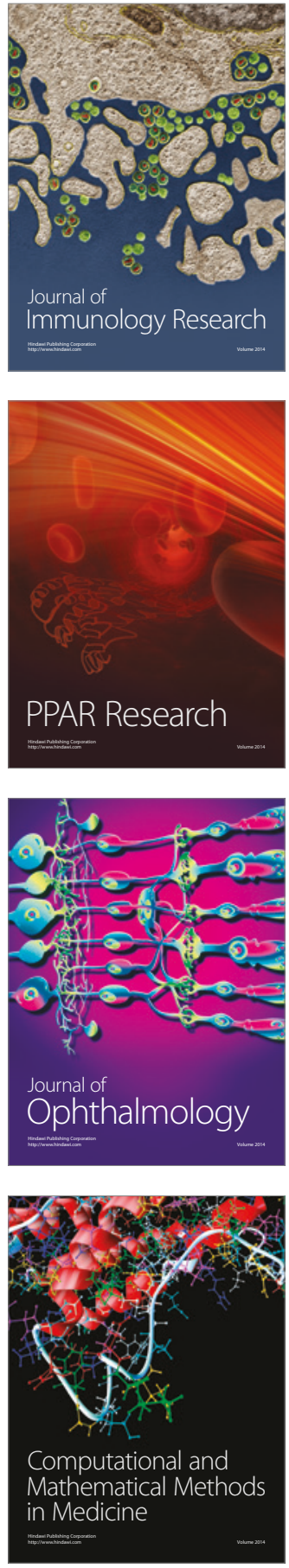

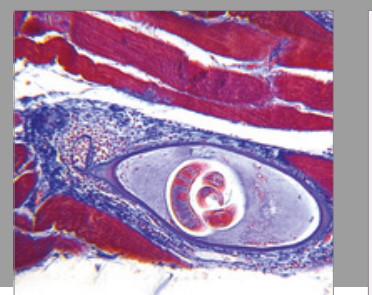

Gastroenterology Research and Practice
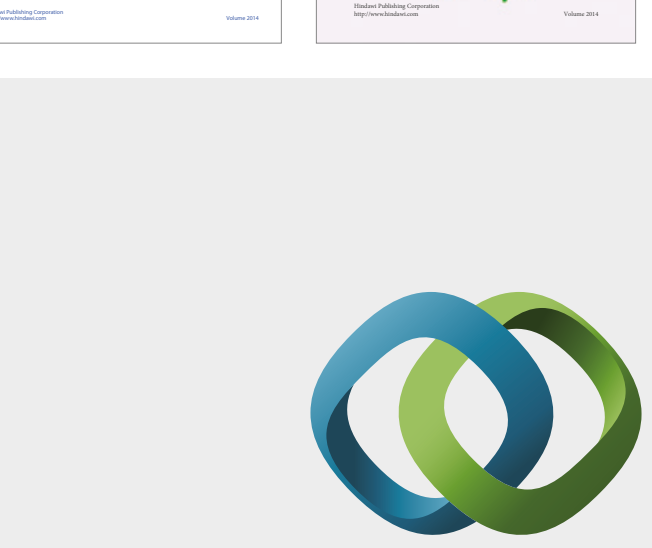

\section{Hindawi}

Submit your manuscripts at

https://www.hindawi.com
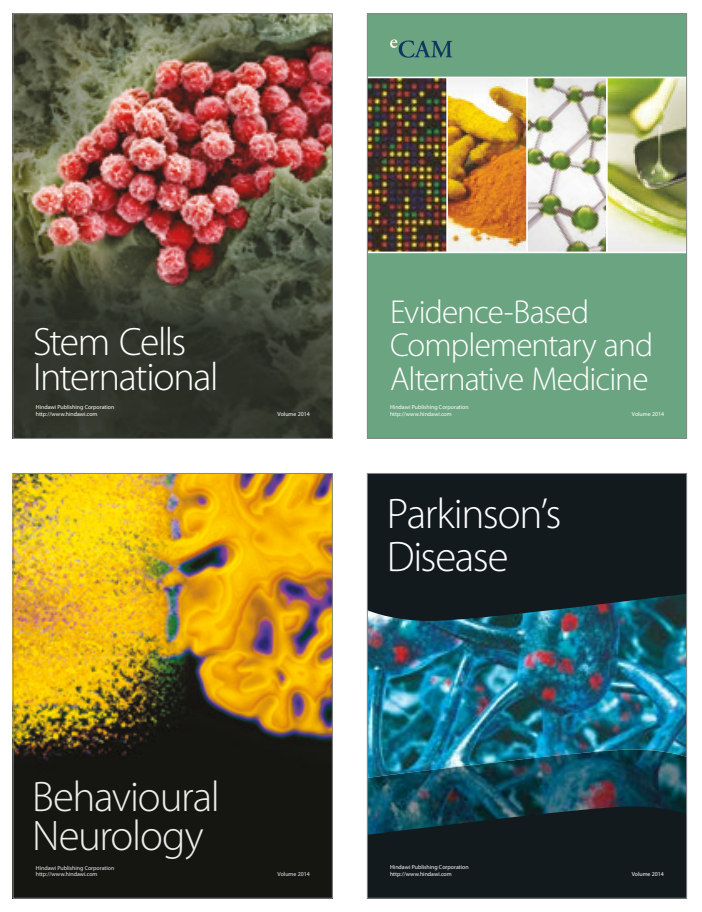
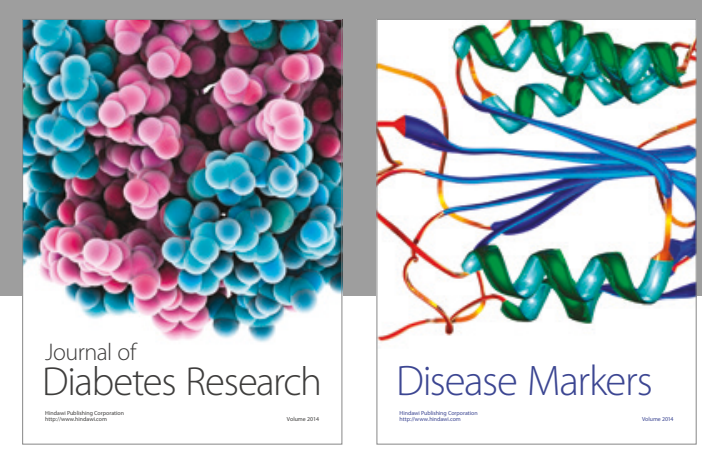

Disease Markers
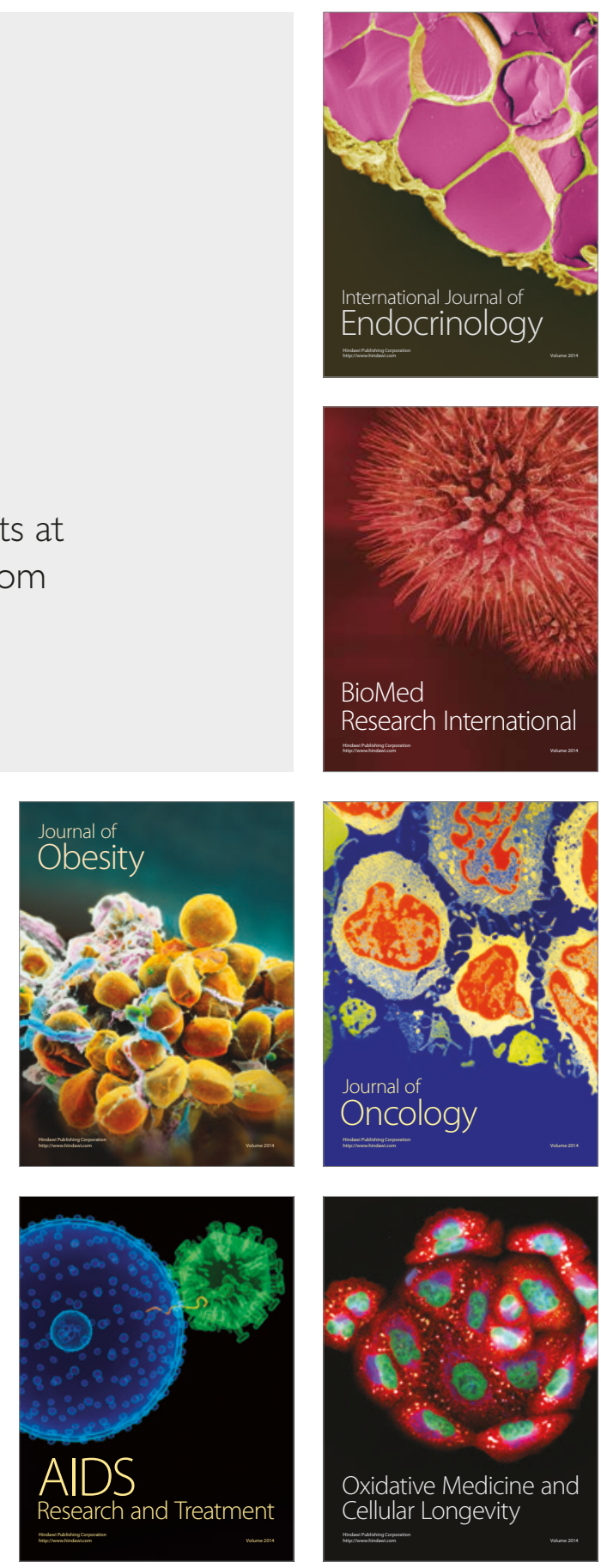